\title{
APPLYING LINEAR SPECTRAL UNMIXING TO AIRBORNE HYPERSPECTRAL IMAGERY FOR MAPPING CROP YIELD VARIABILITY
}

\author{
Chenghai Yang, James H. Everitt, Joe M. Bradford \\ USDA-ARS, Kika de la Garza Subtropical Agricultural Research Center, Weslaco, TX, USA
}

\begin{abstract}
This study evaluated linear spectral unmixing techniques for mapping the variation in crop yield. Both unconstrained and constrained linear spectral unmixing models were applied to airborne hyperspectral imagery recorded from a grain sorghum field and a cotton field. A pair of plant and soil spectra derived from each image was used as endmember spectra to generate unconstrained and constrained plant and soil cover fractions. Yield was positively related to plant fractions and negatively related to soil fractions. For comparison, all 5151 possible narrow-band normalized difference vegetation indices (NDVIs) were calculated from the 102-band images and related to yield. Plant fractions provided better correlations with yield than the majority of the NDVIs. These results indicate that plant cover fraction maps derived from hyperspectral imagery can be used as relative yield maps to characterize crop yield variability.
\end{abstract}

Index Terms - Linear spectral unmixing, hyperspectral imagery, narrow-band NDVI, yield variability

\section{INTRODUCTION}

Multispectral imagery and vegetation indices such as the normalized difference vegetation index (NDVI) have often been used to estimate crop yields and assess within-field yield variability [1-4]. More recently, airborne hyperspectral imagery has been evaluated for mapping crop yield [5-7]. Hyperspectral imagery contains tens to hundreds of narrow bands and has the potential to differentiate and estimate biophysical attributes of interest better than multispectral imagery. However, since hyperspectral imagery has so many bands, it is not always practical to calculate all the possible vegetation indices from these narrow bands. For example, a 102-band hyperspectral image can produce 5151 $[102 ! /(100 ! 2 !)]$ NDVI-type vegetation indices if all possible two-band combinations are considered.

One method to reduce the spectral dimensionality in hyperspectral imagery is to apply stepwise regression analysis to yield data and hyperspectral imagery to identify optimum band combinations for mapping the variation in yield or to use principle components analysis (PCA) and stepwise regression to select the significant principle components that account for most of the variation in yield
[6]. Another method is to calculate various vegetation indices from selected bands [7] or to calculate all possible narrow-band NDVIs [8]. Although stepwise regression can be used to identify the optimum bands for estimating yield, these bands are only the best for the image and yield data from which they are derived and might not be the best for different data sets. Similarly, the optimum narrow-band NDVI identified for one data set might not be the best for another. Therefore, it is necessary to use a technique that can take advantage of the spectral information in all the bands without the need to choose which bands to use.

Spectral unmixing techniques can be used to quantify crop canopy cover within each pixel of an image and have the potential for mapping the variation in crop yield. Each image pixel contains a spectrum of reflectance values for all the wavebands. These spectra can be regarded as the signatures of ground components such as crop plants or soil, provided that a component, referred to as an endmember, occupies the whole pixel. Spectra from mixed pixels can be analyzed with linear spectral unmixing, which models each spectrum in a pixel as a linear combination of a finite number of spectrally pure spectra of the endmembers in the image, weighted by their fractional abundances $[9,10]$.

When linear spectral unmixing is applied to an image, it produces a suite of fraction images, one for each endmember in the model. Each fraction image shows the spatial distribution of the spectrally defined component as an NDVI image does. The fractional abundance of crop plants determined from linear spectral unmixing is a more direct measure of plant cover than an NDVI. Yang et al. [11] applied this technique to hyperspectral imagery for mapping the variation in yield in two grain sorghum fields and found that plant fraction images can be used as relative yield maps. The objectives of this study were to: 1) apply linear spectral unmixing to airborne hyperspectral imagery for estimating plant and soil cover fractions in a grain sorghum field and a cotton field; and 2) relate grain yield to the fractions and compare the correlations with those from all possible narrow-band NDVIs.

\section{METHODS}

Airborne hyperspectral imagery and yield monitor data recorded from a 13.6-ha grain sorghum field in 2000 and a 22.0-ha cotton field in 2001 were used for this study. The 
geographic coordinates near the centers of the sorghum and cotton fields were $\left(98^{\circ} 02^{\prime} 28^{\prime \prime} \mathrm{W}, 26^{\circ} 28^{\prime} 55^{\prime \prime} \mathrm{N}\right)$ and $\left(97^{\circ}\right.$ $\left.58^{\prime} 37^{\prime \prime} \mathrm{W}, 26^{\circ} 29^{\prime} 14^{\prime \prime} \mathrm{N}\right)$, respectively. The soil is dominantly Delfina loamy fine sand. Grain sorghum and cotton are normally grown in rotation.

The imagery was acquired with an airborne hyperspectral imaging system [12]. The system was configured to record imagery with 128 bands from 457.2 to $921.7 \mathrm{~nm}$ at $3.63 \mathrm{~nm}$ intervals. The imagery had a swath width of 640 pixels ( $\sim 1-\mathrm{m}$ pixel size) and a radiometric resolution of 12 bits. Images were acquired on 27 April 2000 from the sorghum field and on 12 June 2001 from the cotton field. At the imaging time, both the sorghum and cotton crops were approaching the maximum canopy cover. The images were corrected for its geometric distortion, rectified to the Universal Transverse Mercator (UTM) coordinate system, and radiometrically calibrated using three reflectance tarpaulins. The last 21 bands and the first 5 bands were removed due to low quantum efficiency near the NIR end of the observed spectrum and the noise in the first few bands of the blue region. The remaining 102 bands with wavelengths from 477.2 to $843.7 \mathrm{~nm}$ were used for analysis. Yield data were recorded with an Ag Leader PF3000 yield monitor (Ag Leader Technology, Ames, Iowa).

Linear spectral unmixing analysis requires the spectra of the endmembers. They can be obtained directly from the image, measured on the ground or derived from a spectral library. In this study, crop plants and bare soil were selected as the relevant endmembers. Thus a simple linear spectral unmixing model has the following form:

$$
y_{i}=a_{i 1} x_{1}+a_{i 2} x_{2}+\varepsilon_{i}, \quad i=1,2, \ldots, n .
$$

where $y_{i}$ is the measured reflectance in band $i$ for a pixel; $a_{i l}$ and $a_{i 2}$ are the known or measured reflectance in band $i$ for plants and soil, respectively; $x_{1}$ and $x_{2}$ are the unknown cover fraction or abundance for plants and soil, respectively; $\varepsilon_{i}$ is the residual between measured and modeled reflectance for band $i$; and $n$ is the number of spectral bands. This model is referred to as the unconstrained linear spectral unmixing model. For constrained linear spectral unmixing, $x_{1}$ and $x_{2}$ should sum to unity.

A pair of plant and soil spectra was extracted from each image to represent pure and healthy plants and bare soil and used as endmember spectra for spectral unmixing analysis for each field. Each endmember spectrum was the average of about 100 pixels identified for the endmember. Both unconstrained and constrained linear spectral unmixing models were applied to each image, and four fractional images (two unconstrained and two constrained) were generated for each field. ENVI (Research Systems, Inc., Boulder, Colorado) was used for this analysis.

Considering the coarse yield data resolutions and positional errors, the 1-m pixel size for the hyperspectral and fraction images was aggregated to $9 \mathrm{~m}$ for sorghum and to $8 \mathrm{~m}$ for cotton. The yield values were similarly averaged from the data points within each larger pixel area. Narrow- band NDVIs were calculated using all 5151 possible twoband combinations of the 102 narrow bands as follows:

$$
\mathrm{NDVI}_{i j}=\left(R_{i}-R_{j}\right) /\left(R_{i}+R_{j}\right),
$$

where $R_{i}$ is the reflectance for band $i, i=1,2, \ldots, n-1$ and $j$ $=i+1, \ldots, n$. Correlation coefficients were calculated among yield, the four fractional images, and the 5151 NDVIs for each field.

\section{RESULTS AND DISCUSSION}

Table 1 gives the univariate statistics of yield and unconstrained and constrained plant and soil fractions derived from the hyperspectral images for the sorghum and cotton fields. Ideally, fraction values should be within the 0 1 range, but in practice they can be negative or exceed 1. For example, the unconstrained plant fraction varies from -0.15 to 1.01 for the sorghum field and from 0.02 to 1.15 for the cotton field. The unconstrained soil fraction varies from 0.02 to 1.16 for sorghum and from -0.21 to 1.12 for cotton. This is because spectral unmixing results can be affected by the purity of the endmembers and the number of endmembers. The linearity assumption of linear spectral unmixing is at best an approximation of the generally nonlinear mixing of surface components.

The mean unconstrained plant fraction is 0.63 for both the sorghum and cotton fields, indicating plant canopy cover was approximately $63 \%$ at the time of the image acquisition. The sum of the plant and soil fractions is 0.95 for sorghum and 0.90 for cotton. Although the unconstrained model does not force the endmember fractions to sum to 1 , the sum is close to 1 , indicating the unconstrained two-endmember linear unmixing model is appropriate for characterizing plant and soil cover in the images. Mean constrained plant fractions are 0.64 for sorghum and 0.60 for cotton and they are similar to the mean unconstrained fractions. Figure 1 shows the unconstrained plant fraction images derived from the hyperspectral images for the two fields. Dark areas have small plant fraction values and represent pixels with a large exposure of soil and sparse plant cover. Conversely, light areas indicate large plant fraction values and represent pixels with dense plant cover.

Table 2 summarizes the correlation coefficients $(r)$ of crop yield with plant and soil fractions for both fields. Yield is positively related to unconstrained and constrained plant fractions, and negatively related to the unconstrained and constrained soil fractions. Unconstrained plant fractions have slightly stronger correlations with yield than the unconstrained soil fractions, whereas constrained plant and soil fractions have identical absolute correlations because they sum to unity. The correlation coefficients for the unconstrained plant fraction are 0.85 for the sorghum field and 0.67 for the cotton field, whereas the r-values for the unconstrained soil fraction are -0.82 for sorghum and -0.61 for cotton. The $r$-values for the constrained plant fraction are 0.85 for sorghum and 0.66 for cotton. 
Table 1. Univariate statistics of crop yield as well as unconstrained and constrained plant and soil fractions derived from the 102-band airborne hyperspectral images for a sorghum field and a cotton field based on a pair of plant and soil endmember spectra extracted from the respective image for each field

\begin{tabular}{lcccc}
\hline $\begin{array}{l}\text { Yield and } \\
\text { endmember fraction }\end{array}$ & Mean & $\begin{array}{c}\text { Standard } \\
\text { deviation }\end{array}$ & Minimum & Maximum \\
\hline $\begin{array}{l}\text { Sorghum field }{ }^{[a]} \text { : } \\
\text { Yield (kg/ha) }\end{array}$ & 3436 & 1480 & 111 & 6022 \\
Unconstrained plant & 0.63 & 0.28 & -0.15 & 1.01 \\
Unconstrained soil & 0.32 & 0.25 & 0.02 & 1.16 \\
Constrained plant & 0.64 & 0.28 & -0.15 & 1.00 \\
Constrained soil & 0.36 & 0.28 & 0.00 & 1.15 \\
& & & & \\
Cotton field: & & & & \\
Yield (kg/ha) & 1404 & 640 & 24 & 3762 \\
Unconstrained plant & 0.63 & 0.22 & 0.02 & 1.15 \\
Unconstrained soil & 0.27 & 0.17 & -0.21 & 1.12 \\
Constrained plant & 0.60 & 0.23 & -0.05 & 1.18 \\
Constrained soil & 0.40 & 0.23 & -0.19 & 1.05 \\
\hline
\end{tabular}

[a] Number of samples was 1661 for the sorghum field and 2150 for the cotton field.
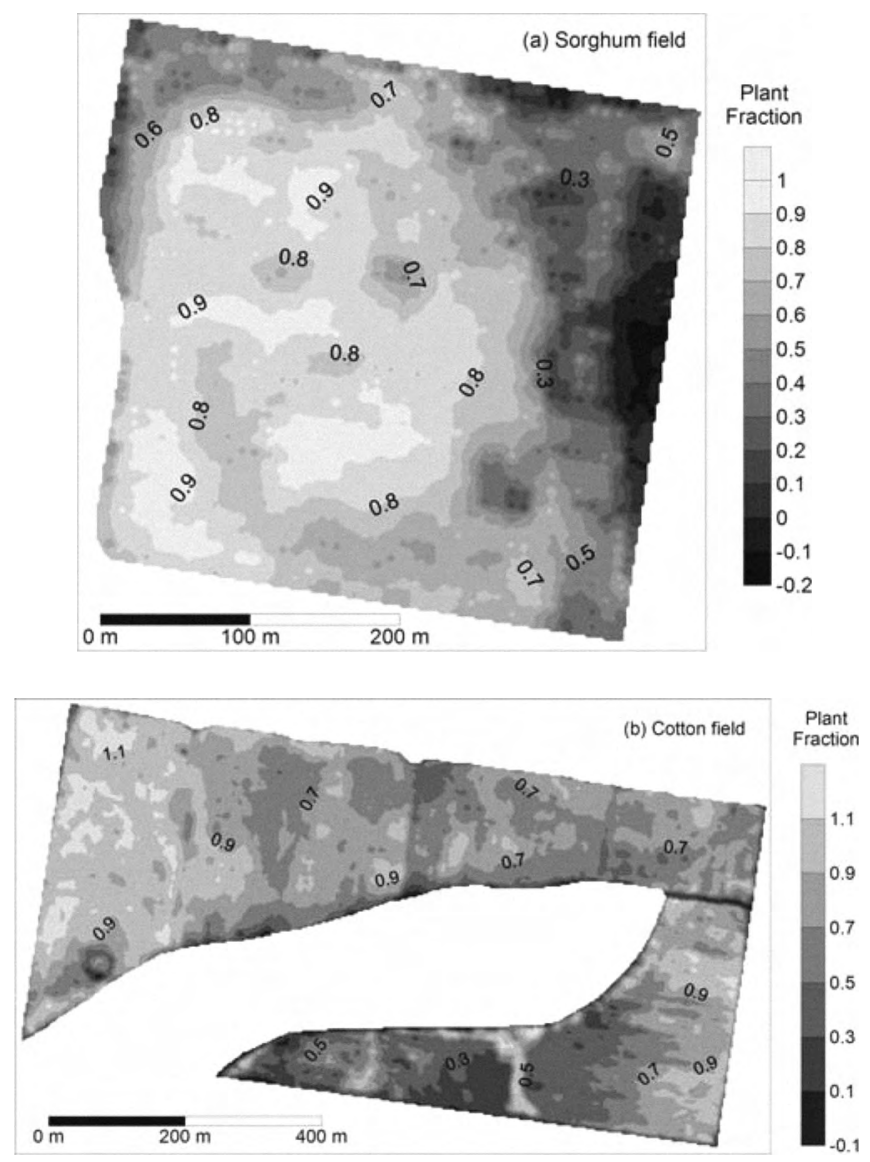

Fig. 1. Unconstrained plant fraction images derived from the 102band airborne hyperspectral images for a sorghum field and a cotton field based on a pair of plant and soil endmember spectra extracted from the image for each field.
Table 2. Correlation coefficients $(r)$ between yield and endmember fractions derived from 102-band airborne hyperspectral images for a sorghum field and a cotton field based on a pair of plant and soil endmember spectra extracted from the respective image for each field

\begin{tabular}{lcc}
\hline Endmember fraction & Sorghum & Cotton \\
\hline Unconstrained plant & $0.85^{[\mathrm{a}]}$ & 0.67 \\
Unconstrained soil & -0.82 & -0.61 \\
Constrained plant & 0.85 & 0.66 \\
Constrained soil & -0.85 & -0.66 \\
\hline
\end{tabular}

[a] All r-values are significant at the 0.0001 level. Number of samples was 1661 for sorghum and 2150 for cotton.

Figure 2 shows the contour maps of absolute $r$-values between yield and all 5151 possible NDVIs for both the sorghum and cotton fields. The absolute $r$-values vary from 0 to 0.88 for sorghum and from 0 to 0.72 for cotton. The $r$ values are generally larger when one band has wavelengths smaller than $730 \mathrm{~nm}$ and the other band has wavelengths larger than $730 \mathrm{~nm}$. However, the best r-values $(>0.85)$ occur when one band is around $730 \mathrm{~nm}$ and the other was over $760 \mathrm{~nm}$ for the sorghum field. Also large $r$-values $(>0.825)$ for the sorghum field occur when one band in a pair has wavelengths between $550 \mathrm{~nm}$ and $575 \mathrm{~nm}$ and the other has wavelengths between $575 \mathrm{~nm}$ and $690 \mathrm{~nm}$. Based on the contour maps of $r$-values, better NDVI images are more likely to be obtained by selecting one band in the visible region and the other in the NIR region.

In comparison, the best NDVIs have larger correlations with yield than the best fraction images for both fields. Nevertheless, the best fraction-based $r$-values $(0.85$ for sorghum and 0.67 for cotton) are better than $97.1 \%$ and $96.0 \%$ of the 5151 NDVI-based $r$-values for the sorghum field and the cotton field, respectively. If the objective of a study is to determine the best correlation based on actual yield data, all possible narrow-band NDVIs should be derived to identify the best NDVI. However, if the objective is to generate a spectral map from a hyperspectral image to characterize the spatial variation in yield without knowing the actual yield, an unconstrained plant fraction image based on a pair of plant and soil spectra will be a better choice. An unconstrained plant fraction image appears to provide a better relative yield map than an NDVI image derived from two randomly selected bands. An NDVI image uses only two narrow bands, whereas a plant fraction image is based on all bands in the image. Although an NDVI image could provide better $r$-values than a plant fraction image as shown in this study, the best NDVI identified from one image is unlikely to be the best NDVI for another. Moreover, the best NDVI can only be identified if the yield is known and all possible NDVIs are calculated. On the other hand, the unconstrained plant fraction image can be generated using all the bands and a pair of plant and soil endmember spectra without the need to know yield information.

If yield data are not available or if the user does not have the time or capability to derive all NDVIs, the 
unconstrained plant fraction can be used as a relative yield map. If yield data are available, all NDVIs can be calculated to determine the best one for mapping yield. In this case, the unconstrained plant fraction can also be used as an alternative because it has the potential to be as good as or even better than the best NDVI. Therefore, this technique can be used alone or in conjunction with other traditional VIs in mapping yield variability and other applications.
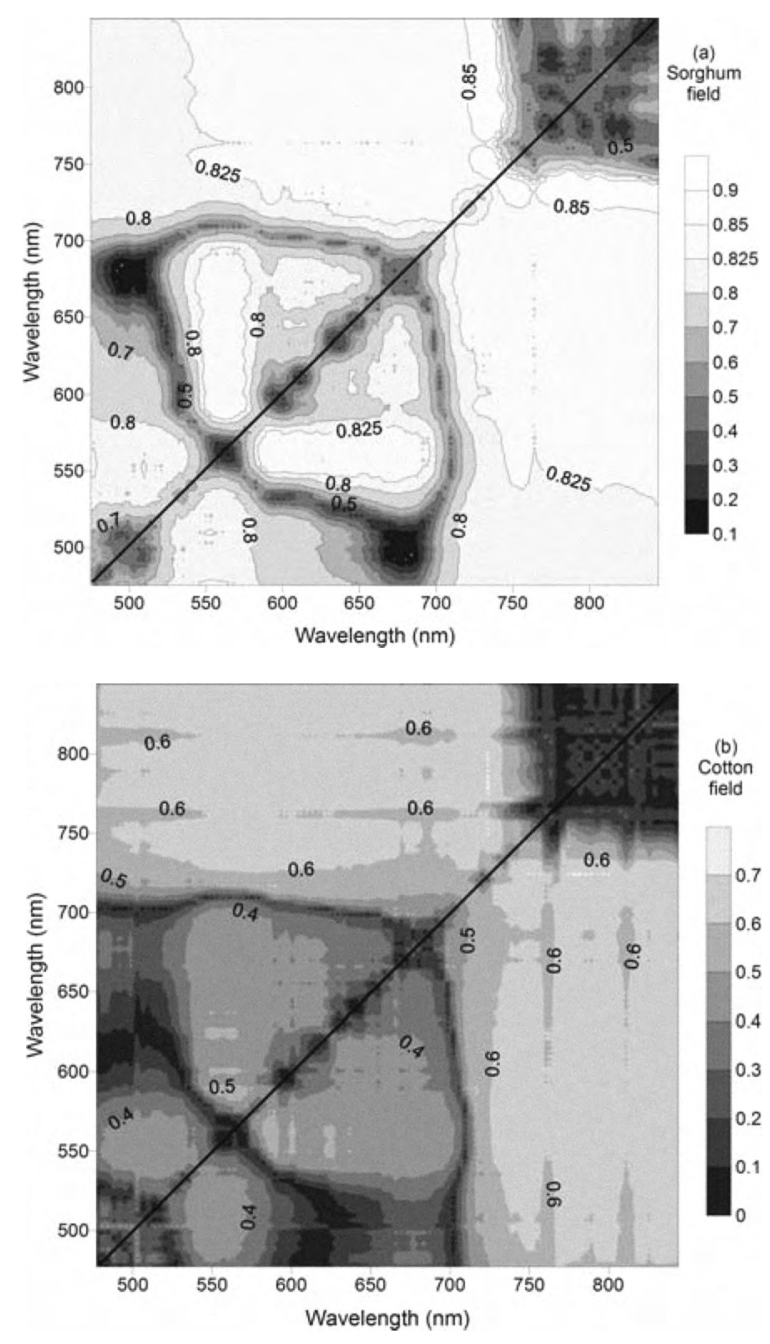

Fig. 2. Contour maps showing absolute correlation coefficients between crop yield and all possible narrow-band NDVIs derived from 102-band airborne hyperspectral images for a sorghum field and a cotton field. When band $i=$ band $j, \mathrm{NDVI}_{i j}=0$ and there are no correlations (shown by the diagonal line).

\section{CONCLUSIONS}

This study demonstrates the use of linear spectral unmixing techniques to derive plant and soil fractions from hyperspectral imagery for mapping the variation in yield for both grain sorghum and cotton. The unconstrained plant fraction provides better $r$-values with yield than most of the 5151 narrow-band NDVIs derived from the 102-band hyperspectral images for both crops. In practice, an unconstrained plant fraction image can be a better representation of relative yield than an NDVI image derived from two bands if no yield sampling data are available.

\section{REFERENCES}

[1] G.B. Senay, A.D. Ward, J.G. Lyon, N.R. Fausey, and S.E. Nokes, "Manipulation of high spatial resolution aircraft remote sensing data for use in site-specific farming," Transactions of the ASAE 41(2): 489-495, 1998.

[2] C. Yang, and J.H. Everitt, "Relationships between yield monitor data and airborne multispectral multidate digital imagery for grain sorghum," Precision Agriculture 3(4): 373-388, 2002.

[3] A. Dobermann, and J. L. Ping, "Geostatistical integration of yield monitor data and remote sensing improves yield maps," Agronomy Journal 96: 285-297, 2004

[4] D. Inman, R. Khosla, R. Reich, and D.G. Westfall, "Normalized difference vegetation index and soil color-based management zones in irrigated maize," Agronomy Journal 100: 6066, 2008.

[5] P.K. Goel, S.O. Prasher, J.A. Landry, R.M. Patel, A.A. Viau, and J.R. Miller, "Estimation of crop biophysical parameters through airborne and field hyperspectral remote sensing," Transactions of the ASAE 46(4): 1235-1246, 2003

[6] C. Yang, J.H. Everitt, J.M. Bradford, "Airborne hyperspectral imagery and yield monitor data for estimating grain sorghum yield variability," Transactions of the ASAE 47(3): 915-924, 2004.

[7] P.J. Zarco-Tejada, S.L. Ustin, and M.L. Whiting, "Temporal and spatial relationships between within-field yield variability in cotton and high-spatial hyperspectral remote sensing imagery," Agronomy Journal 97: 641-653, 2005.

[8] P.S. Thenkabail, R.B. Smith, and E.D. Pauw, "Hyperspectral vegetation indices and their relationships with agricultural crop characteristics," Remote Sensing of Environment 71: 158-182, 2000 .

[9] J.B. Adams, M.O. Smith, and P.E. Johnson, "Spectral mixture modeling: A new analysis of rock and soil types at the Viking Lander 1 site," Journal of Geophysical Research 91: 8098-8112, 1986.

[10] M.O. Smith, S.L. Ustin, J.B. Adams, and A.R. Gillespie, "Vegetation in deserts: I. A regional measure of abundance from multispectral images," Remote Sensing of Environment 31: 1-26, 1990.

[11] C. Yang, J.H. Everitt, and J.M. Bradford, "Airborne hyperspectral imagery and linear spectral unmixing for mapping variation in crop yield," Precision Agriculture 8(6): 279-296, 2007.

[12] C. Yang, J.H. Everitt, M.R. Davis, and C. Mao, "A CCD camera-based hyperspectral imaging system for stationary and airborne applications," Geocarto International 18(2): 71-80, 2003. 